\title{
ASSOCIATED BASIC HYPERGEOMETRIC SERIES
}

by R. P. AGARWAL

(Received 22nd May, 1953)

1. Introduction. The purpose of the present note is to give some interesting and simple identities connected with basic hypergeometric series of the types ${ }_{2} \Phi_{1}$ and ${ }_{3} \Phi_{2}$.

The difference operator

$$
D f(x) \equiv \frac{f(x)-f(q x)}{x}, \quad(q=1-\epsilon, \epsilon>0)
$$

is of much importance in the theory of basic hypergeometric functions and has been used by many authors : e.g., Heine (1), Rogers (2), Jackson (3) and Hahn (4), etc., in developing the theory of basic functions. The operator, $D$ in the theory of basic functions replaces the ordinary differential operator $d / d x$.

In $\S 3$, I use this operator to obtain some identities involving the function ${ }_{2} \Phi_{1}$. In $\S 4$, a basic generalisation of Gauss's theorem (extended by Riemann), that any three series of the ordinary hypergeometric type $F(a+l, b+m ; c+n ; x)$, where $l, m, n$ are integers (positive or negative) are connected by a linear homogeneous relation with polynomial coefficients, is given.

2. Notation. Let

and

$$
\begin{aligned}
& (a)_{n}=\left(1-q^{a}\right)\left(1-q^{a+1}\right) \ldots\left(1-q^{a+n-1}\right), \quad|q|<1, \\
& (a)_{0}=1,
\end{aligned}
$$

$$
{ }_{s+1} \Phi_{s}\left(\begin{array}{l}
a_{1}, \ldots, a_{s+1} ; \dot{x} \\
b_{1}, \ldots, b_{s}
\end{array}\right)=\sum_{n=0}^{\infty} \frac{\left(a_{1}\right)_{n} \ldots\left(a_{s+1}\right)_{n}}{(1)_{n}\left(b_{1}\right)_{n} \ldots\left(b_{s}\right)_{n}} x^{n}
$$

Also, for the sake of brevity, we will use the notation

$$
\begin{aligned}
& \alpha \equiv\left(q^{-a}-1\right), \quad \beta \equiv\left(q^{-b}-1\right), \quad \gamma \equiv\left(q^{-c}-1\right), \\
& \delta \equiv\left(q^{-d}-1\right) \text { and } \epsilon \equiv\left(q^{-e}-1\right)
\end{aligned}
$$

3. We now prove the following identities :

(i) $(a)_{n} x^{a-1}{ }_{2} \Phi_{1}(a+n, b ; c ; x)=D^{n}\left[x^{a+n-1}{ }_{2} \Phi_{1}(a, b ; c ; x)\right]$,

(ii) $(c-n)_{n} x^{c-1-n} \Phi_{1}(a, b ; c-n ; x)=D^{n}\left[x^{c-1} \Phi_{1}(a, b ; c ; x)\right]$,

(iii) $(a)_{n}(b)_{n_{2}} \Phi_{1}(a+n, b+n ; c+n ; x)=(c)_{n} D^{n}\left[{ }_{2} \Phi_{1}(a, b ; c ; x)\right]$,

(iv) $(c-a)_{n} x^{c-a-1} \prod_{m=0}^{\infty} \frac{\left(1-x q^{c-a-b+n+m}\right)}{\left(1-x q^{m}\right)}{ }_{2} \Phi_{1}\left(a-n, b ; c ; x q^{c-a-b+n}\right)$

$$
=D^{n}\left[x^{c-a+n-1} \prod_{m=0}^{\infty} \frac{\left(1-x q^{c-a-b+m}\right)}{\left(1-x q^{m}\right)}{ }_{2} \Phi_{1}\left(a, b ; c ; x q^{c-a-b}\right)\right]
$$

(v) $(c-n)_{n} x^{c-1-n} \prod_{m=0}^{\infty} \frac{\left(1-x q^{c-a-b+n+m}\right)}{\left(1-x q^{m}\right)}{ }_{2} \Phi_{1}\left(a-n, b-n ; c-n ; x q^{c-a-b+n}\right)$

$$
=D^{n}\left[x^{c-1} \prod_{m=0}^{\infty} \frac{\left(1-x q^{c-a-b+m}\right)}{\left(1-x q^{m}\right)}{ }_{2} \Phi_{1}\left(a, b ; c ; x q^{c-a-b}\right)\right]
$$


(vi) $(c-a)_{n}(c-b)_{n} \prod_{m=0}^{\infty} \frac{\left(1-x q^{c-a-b+n+m}\right)}{\left(1-x q^{m}\right)}{ }_{2} \Phi_{1}\left(a, b ; c+n ; x q^{c-a-b+n}\right)$

$$
=(c)_{n} D^{n}\left[\prod_{m=0}^{\infty} \frac{\left(1-x q^{c-a-b+m}\right)}{\left(1-x q^{m}\right)}{ }_{2} \Phi_{1}\left(a, b ; c ; x q^{c-a-b}\right)\right] \text {. }
$$

To prove the first three we expand the right-hand ${ }_{2} \Phi_{1}$ in powers of $x$ and use the relation term by term.

$$
D x^{a}=\left(1-q^{a}\right) x^{a-1}
$$

The last three are variants of the first three in order. They are obtained from the first three by using the well-known transformation

$$
{ }_{2} \Phi_{1}(a, b ; c ; x)=\prod_{n=0}^{\infty} \frac{\left(1-x q^{a+b-c+n}\right)}{\left(1-x q^{n}\right)}{ }_{2} \Phi_{1}\left(c-a, c-b ; c ; x q^{a+b-c}\right)
$$

on both sides of (i), (ii) and (iii) respectively to transform the ${ }_{2} \Phi_{1}$. The identity (v) is the basic analogue of the well-known result due to Jacobi (5) for the ordinary hypergeometric function.

4. In this section I will generalise Gauss's* theorem for ordinary hypergeometric associated series by showing that between any four series of the type

$$
{ }_{3} \Phi_{2}\left[\begin{array}{c}
a+l, b+m, c+n ; x \\
d+p, e+s
\end{array}\right]
$$

where $l, m, n, p$ and $s$ are integers (positive or negative), there always exists a linear homogeneous relation with polynomial coefficients.

To prove this we can easily verify that the difference equation satisfied by

is

$$
{ }_{3} \Phi_{2}(a, b, c ; d, e ; x)
$$

$$
\left\{\vartheta\left(\vartheta+q^{1-d}-1\right)\left(\vartheta+q^{1-\ell}-1\right)-x q^{a+b+c-a-e+2}(\vartheta+\alpha)(\vartheta+\beta)(\vartheta+\gamma)\right\} \Phi=0,
$$

where $\vartheta \equiv x D$.

Also, it is easily verified that

and

$$
(\vartheta+\alpha) \Phi=\alpha \Phi_{a+}
$$

$$
\left(q^{1-e}-1\right) \Phi_{e^{-}}=\left(\vartheta+q^{1-e}-1\right) \Phi,
$$

where $\Phi$ denotes the function ${ }_{3} \Phi_{2}$ and

$$
\Phi_{a+}={ }_{3} \Phi_{2}\left[\begin{array}{c}
a+1, b, c ; x \\
d, e ;
\end{array}\right]
$$

with similar abbreviated notations for other associated series.

Now (4.1), with $a-1$ in place of $a$, can be written as

$$
\begin{array}{r}
\left\{\vartheta^{2}+\vartheta\left(q^{1-a}+q^{1-e}-q^{1-a}-1\right)+q^{2}(\epsilon-\alpha)(\delta-\alpha)-x q^{a+b+c-d-\epsilon+1}(\vartheta+\beta)(\vartheta+\gamma)\right\}\left(\vartheta+q^{1-a}-1\right) \Phi_{a-} \\
=q^{2}(\epsilon-\alpha)(\delta-\alpha)\left(q^{1-a}-1\right) \Phi_{a-} \ldots \ldots \ldots \ldots \ldots \ldots \ldots \ldots \ldots \ldots \ldots \ldots \ldots \ldots \ldots \ldots \ldots \ldots \ldots \ldots \ldots \ldots \ldots \ldots \ldots \ldots \ldots
\end{array}
$$

Using (4.2) with $a-1$ in place of $a$ we get

$$
\begin{aligned}
q^{2}(\epsilon-\alpha)(\delta-\alpha) \Phi_{a-}=\left\{\vartheta^{2}+\vartheta\left(q^{1-d}+q^{1-e}-q^{1-a}-1\right)\right. & +q^{2}(\epsilon-\alpha)(\delta-\alpha)-x q^{a+b+c-d-e+1} \\
& \times(\vartheta+\beta)(\vartheta+\gamma)\} \Phi .
\end{aligned}
$$

* For similar results for ordinary hypergeometric series see Bailey, Quart. J. of Math., Oxford, 8 (1937), pp. 115-118. 
Next, replacing $e$ by $e+1$ in (4.1) and proceeding as above, we get, on using (4.3) $x q^{1-d-e+a+b+c}(\alpha-\epsilon)(\beta-\epsilon)(\gamma-\epsilon) \Phi_{e+}$ $=\left\{\vartheta\left(\vartheta+q^{1-d}-1\right)-x q^{a+b+c-d-e+1}\left(\vartheta^{2}+\vartheta(\alpha+\beta+\gamma-\epsilon)+\alpha(\beta-\epsilon)+\beta(\gamma-\epsilon)+\gamma(\alpha-\epsilon)+\epsilon^{2}\right)\right\} \epsilon \Phi$.

Now, by repeated applications of the relations (4.2), (4.3), (4.5) and (4.6) and similar other relations, together with the use of the equation (4.1), we can express any associated series

$$
{ }_{3} \Phi_{2}\left[\begin{array}{c}
a+l, b+m, c+n ; x \\
d+p, e+s
\end{array}\right]
$$

in terms of $\Phi, \vartheta \Phi$ and $\vartheta^{2} \Phi$. Thus between any four relations of this type we can eliminate $\Phi, \vartheta \Phi$ and $\vartheta^{2} \Phi$ to get a linear homogeneous relation between four associated series of the type ${ }_{3} \Phi_{2}$, with polynomial coefficients.

\section{REFERENCES}

(1) Heine, E., Theorie der Kugelfunctionen, I. (1878), pp. 97-125.

(2) Rogers, L. J., Proc. Lond. Math. Soc. (1), 24 (1893), pp. 337-352.

(3) Jackson, F. H., Quart. Journ. Math. (Oxford) (2), 2 (1951), pp. 1-16.

(4) Hahn, W., Math. Nachrichten, 3 (1950), pp. 257-294.

(5) Jacobi, C. G. J., Werke, VI., pp. 191-193.

\section{Mathematics Department}

BEDFORD COLLEGE

London, N.W.1. 